\title{
HIGH ORDER SLIDING MODE CONTROL WITH ANTI-SWAY BASED COMPENSATION ON ARTIFICIAL NEURAL NETWORK BY PSO ALGORITHM FOR OVERHEAD CRANE
}

\author{
Le Xuan Hai ${ }^{1,}$, Nguyen Van Thai ${ }^{1}$, Vu Thi Thuy Nga ${ }^{1}$, Hoang Thi Tu Uyen ${ }^{1}$, \\ Nguyen Thanh Long ${ }^{1}$, Thai Huu Nguyen ${ }^{2}$, Phan Xuân Minh ${ }^{1}$ \\ ${ }^{1}$ Hanoi University of Science and Technology, No 1, Dai Co Viet Street, \\ Hai Ba Trung District, Ha Noi City \\ ${ }^{2}$ Vinh University of Technology Education, Hung Dung Ward, Vinh City, Nghe An Province \\ *Email: xhaicuwc.edu.vn@gmail.com
}

Received: 15 August 2016; Accepted for publication: 2 March 2017

\begin{abstract}
This paper proposes a second order sliding mode controller combined with signal set calibrator for overhead crane tracking desired position and resisting disturbance. High order sliding mode controller ensures that the overhead crane tracks desired trajectory and resists disturbance. Neural network is trained by particle swarm optimization algorithm (PSO) to compensate anti-sway for load. The results on the computer simulation show that high order sliding mode controller with anti-sway compensation for overhead crane tracks desired trajectory and the swing of load that is smaller than high order sliding mode controller without anti-sway compensation.
\end{abstract}

Keywords: high order sliding mode control, artificial neural network, particle swarm optimization algorithm (PSO), anti-sway for overhead crane.

\section{PROBLEM STATEMENT}

Overhead crane is one of the essential equipment that are commonly used in industrial factory, harbors for transporting heavy goods and it is also researching object recently. Mathematical model of overhead crane is categorized as under-actuated robot.

The solution to track desired trajectory of trolley and anti-sway of load are particular characteristics of overhead crane. The approaches for anti-sway are based on PD techniques control [1], partial feedback linearization control [2, 3], nonlinear control [4 - 11], robust adaptive control [12 - 14], fuzzy - neural network controller [15, 16]. The above controllers are often used for uncertainly parameters of overhead crane and when executed to combine with two loop circuits : the adaptive parameters adjustment loop and control loop. These controllers generally have complex structures when in fact implemented to select the right device, it is not always easy. 
Therefore, in this paper, we proposed high order sliding mode controller with optimal trajectory to reduce swing angle of load when moving process to desired position. Optimal trajectory is generated by Radial Basis Function Neural (RBFNs) Networks that is trained by PSO algorithm. Thus, structure control contains a high order sliding mode controller and an antisway compensator by RBFNs.

\section{OVERHEAD CRANE MODEL}

The model of overhead crane is shown in Figure 1. The trolley is moved by $F$ force. The motion of load is always on $X-Y$ plane.

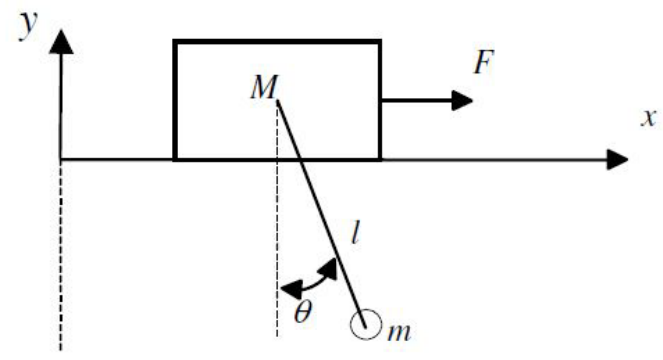

Figure 1. Overhead crane model.

Assuming that the trolley and the load can be regarded as point mass, friction force in trolley can be neglected . Overhead crane model is expressed as:

$$
\begin{aligned}
& (M+m) \ddot{x}+m l \ddot{\theta} \cos \theta-m l \dot{\theta}^{2} \sin \theta=F \\
& l \ddot{\theta}+g \sin \theta+\ddot{x} \cos \theta=0
\end{aligned}
$$

where: $x, l$ and $\theta$ are trolley position, length of suspension rope and swing angle of load, respectively. Defining $u=F$ and state vector $X^{T}=\left[\begin{array}{lllll}x_{1} & x_{2} & x_{3} & x_{4}\end{array}\right]^{T}=\left[\begin{array}{llll}x & \dot{x} & \theta & \dot{\theta}\end{array}\right]^{T}$. The equation (1) is written in the form of state space model as the following:

$$
\begin{aligned}
& \dot{x}_{1}=x_{2} \\
& \dot{x}_{2}=f_{1}(X)+g_{1}(X) u \\
& \dot{x}_{3}=x_{4} \\
& \dot{x}_{4}=f_{2}(X)+g_{2}(X) u
\end{aligned}
$$

where:

$$
\begin{array}{ll}
f_{1}(X)=\frac{m l \dot{\theta}^{2} \sin \theta+m g \sin \theta \cos \theta}{M+m \sin ^{2} \theta} & g_{1}(X)=\frac{1}{M+m \sin ^{2} \theta} \\
f_{2}(X)=-\frac{(M+m) g \sin \theta+m l \dot{\theta}^{2} \sin \theta \cos \theta}{\left(M+m \sin ^{2} \theta\right) l} & g_{2}(X)=-\frac{\cos \theta}{\left(M+m \sin ^{2} \theta\right) l}
\end{array}
$$

So, the model of overhead crane is divided into two subsystems: the positioning subsystem and anti-swing subsystem. The purpose of the controller designation is to keep the trolley tracking the reference trajectory without sway of load under the condition of disturbance.

\section{SECOND ORDER SLIDING MODE CONTROL}

Defining tracking error vector: 


$$
e(t)=\left[\begin{array}{l}
x_{1}-x_{d} \\
x_{3}-\theta_{d}
\end{array}\right]=\left[\begin{array}{l}
x-x_{d} \\
\theta-\theta_{d}
\end{array}\right]=\left[\begin{array}{l}
e_{1} \\
e_{3}
\end{array}\right]
$$

where : $x_{d}$ and $\theta_{d}$ are desired trajectory and swing angle of load, respectively. Of course, the desired swing angle of load is zero. Assuming that the first and the second time derivative of $x_{d}$ are determined and uniformly bounded, the equation (2) is transferred to error model:

$$
\begin{aligned}
& \dot{e}_{1}=e_{2} \\
& \dot{e}_{2}=f_{1}(X)+g_{1}(X) u-\ddot{x}_{d} \\
& \dot{e}_{3}=e_{4} \\
& \dot{e}_{4}=f_{2}(X)+g_{2}(X) u
\end{aligned}
$$

Defining sliding surface for each subsystem as :

$$
\begin{aligned}
& s_{1}=c_{1} e_{1}+e_{2} \\
& s_{2}=c_{2} e_{3}+e_{4}
\end{aligned}
$$

Then, the second order sliding surface is defined:

$$
s=\alpha s_{1}+\beta s_{2}
$$

where: $c_{1}, c_{2}, \alpha$ and $\beta$ are positive constants. In order to make the close-loop system that has sliding surface $s$ is asymptotic stability, the following condition should be satisfied:

$$
\dot{s}=-k_{1} \operatorname{sgn}(s)-k_{2} s=\alpha \dot{s}_{1}+\beta \dot{s}_{2}
$$

This leads to the control signal $u$ :

$$
u=-\frac{\alpha f_{1}(X)+\beta f_{2}(X)+\alpha c_{1} e_{2}+\beta c_{2} e_{4}-\alpha \ddot{x}_{d}+k_{1} \operatorname{sgn}(s)+k_{2} s}{\alpha g_{1}(X)+\beta g_{2}(X)} .
$$

\section{COMPENSATION BY USING ARTIFICIAL NEURAL NETWORK}

\subsection{Neural network structure}

Artificial is used to generate optimal trajectory of the trolley from the initial position to desired position in time TE and reduce the sway of load.

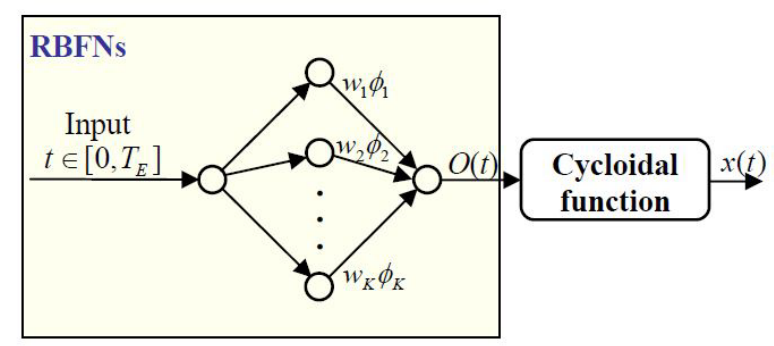

Figure 2. Radial Basis Function Neural Networks.

Figure 2 shows neural network structure that is used in this paper. As shown in Figure 2, RBFNs consist of an input, $K$ neurals in the hidden layer, and an output layer. The input layer 
to RBFNs is given by values of time from 0 to TE. The output of the k-th neural of the hidden layer is expressed by the Gaussian function as:

$$
\phi_{k}(t)=\exp \left\{-\frac{\left(t-c_{k}\right)^{2}}{\sigma_{k}^{2}}\right\},(k=1,2, \ldots, K)
$$

where: $c_{k}$ and $\sigma_{k}$ are center and radius, respectively. The output of RBFNs is calculated by:

$$
\phi_{k}(t)=\exp \left\{-\frac{\left(t-c_{k}\right)^{2}}{\sigma^{2}{ }_{k}}\right\},(k=1,2, \ldots, K)
$$

where: $w_{k}$ is weight between the hidden layer and output layer.

The trajectory of the trolley requires that both velocity and acceleration be equal to zero at the start and the end point. So, the following constraint conditions are applied for trajectory of trolley:

$$
\dot{x}(0)=\dot{x}(T E)=\ddot{x}(0)=\ddot{x}(T E)=0
$$

using the cycloidal function to satisfy above condition:

$$
\Phi(u)=X E\left\{u-\frac{\sin (2 \pi u)}{2 \pi}\right\}
$$

The position of the trolley is generated as :

$$
x(t)=\Phi\{O(t)\}
$$

Equation (11) determines that the output of RBFNs is input $u$ of Cycloidal (10). Moreover, natural trajectory of the trolley satisfies following condition:

$$
x(0)=0, \quad x(T E)=X E
$$

Therefore, the following condition is required for output of the RBFNs:

$$
O(0)=0, \quad O(T E)=1
$$

In order to satisfy condition (13), the weights $w_{k}$ and $w_{k-1}$ are determined by the following equation system:

$$
\left\{\begin{array}{l}
\sum_{k=1}^{K-2} w_{k} \phi_{k}(0)+w_{K-1} \phi_{K-1}(0)+w_{K} \phi_{K}(0)=0 \\
\sum_{k=1}^{K-2} w_{k} \phi_{k}(T E)+w_{K-1} \phi_{K-1}(T E)+w_{K} \phi_{K}(T E)=1
\end{array}\right.
$$

\subsection{PSO algorithm}

In this part, the PSO algorithm will be introduced to train RBFNs, $\sigma_{1}, \sigma_{2}, \ldots, \sigma_{K} \sigma_{1}, \sigma_{2}, \ldots, \sigma_{K}$ and $w_{1}, w_{2}, \ldots, w_{K-2}$ are changed. By this way, the trajectory of the trolley is optimal and the sway of load is removed.

To have minimum swing angle, the function $f=\theta^{2}+\dot{\theta}^{2}$ (with $\theta$ is swing angle after time $\mathrm{TE}$ ) is defined as objective function that need to optimize.

The algorithm for trajectory generation based on the PSO is summarized as follows: 
Step 1: The positions and velocities of all particles are initialized randomly. The position and velocity of $i-$ th particle are defined as :

$$
x_{i}=\left[\begin{array}{llll}
x_{i, 1} & x_{i, 2} & \ldots & x_{i, d}
\end{array}\right], v_{i}=\left[\begin{array}{llll}
v_{i, 1} & v_{i, 2} & \ldots & v_{i, d}
\end{array}\right]
$$

where: $d=3 K-2$

Step 2: Calculate $w_{K-1}$ and $w_{K}$ from (14), then the reference position is obtained from (11). Next, the value of $f$ is calculated from the second equation of (10). So, the initial value of $f$ of each particle is determined.

Step 3: Initial value of pbest $_{i}$ is initial position of $i$-th particle. In swarm, we determine the particle that has best position as gbest .

Step 4: Velocity and position of each partial are updated as following equations:

$$
\begin{aligned}
& v_{i}^{(n+1)}=\chi\left[v_{i}^{(n)}+a_{1} r_{1}^{(n)}\left(\text { pbest }_{i}-x_{i}^{(n)}\right)+a_{2} r_{2}^{(n)}\left(\text { gbest }-x_{i}^{(n)}\right)\right] \\
& x_{i}^{(n+1)}=x_{i}^{(n)}+v_{i}^{(n+1)}
\end{aligned}
$$

where $n$ is iteration number, $r_{1}$ and $r_{2}$ are two independent uniform random numbers with values from 0 to $1 . \quad \chi$ is defined as:

$$
\chi=\frac{2}{\left|2-\phi-\sqrt{\phi^{2}-4 \phi}\right|}, \quad \phi=a_{1}+a_{2}, \quad \phi>4
$$

Typically, $a_{1}=a_{2}=2.05$.

Step 5: Calculate $f$ value of each particle using the same procedure as that described in Step 2. For each particle, if current position is better than pbest, pbest takes current position. For all swarm, gbest takes the best value in all pbest value.

Step 6: If $n$ is less than maximum iteration number, $n=n+1$ and Step $4 \rightarrow 6$ are repeated. Otherwise, gbest is optimal position.

\subsection{High level sliding mode control based on anti-sway system}

The structure of over all system is shown in Figure 3. In this system, the desired trajectory is gotten from NBFNs then fed to sliding mode controller. With this combination, the operation of the over head crane system not only to track the reference trajectory but also to exclude the effect of the disturbance and reduces the oscillation of the load during the movement of the trolley.

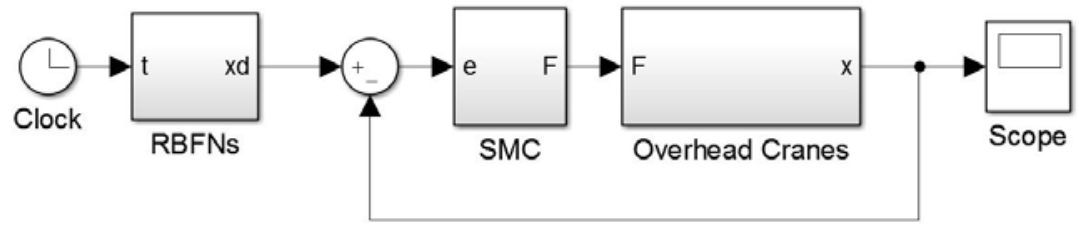

Figure 3. Sliding mode control combined compensation anti-sway based on artificial neural network by PSO algorithm system structure. 


\section{NUMERICAL SIMULATIONS}

In this part, a simulation based on Matlab/SIMULINK is executed to verify the effectiveness of the proposed algorithm. The parameters of overhead crane are as follows: $M=5 \mathrm{~kg}, m=2.5 \mathrm{~kg}, l=1 \mathrm{~m}$ and $g=9.81 \mathrm{~m} / \mathrm{s}^{2}$. The disturbance is occurred suddenly at 1 second: $d_{1}(t)=d_{2}(t)=1(t-1)-1(t-1.1)$.

The parameters of sliding mode controller are selected as : $c_{1}=2, c_{2}=0.2, \alpha=4, \beta=4$, $k_{1}=3.8, k_{2}=3.5$.

The parameters for PSO algorithm are: $T E=5, X E=2$, maximum iteration number is 50, swarm has 20 particles and hidden layer has 10 neurals $(K=10)$.

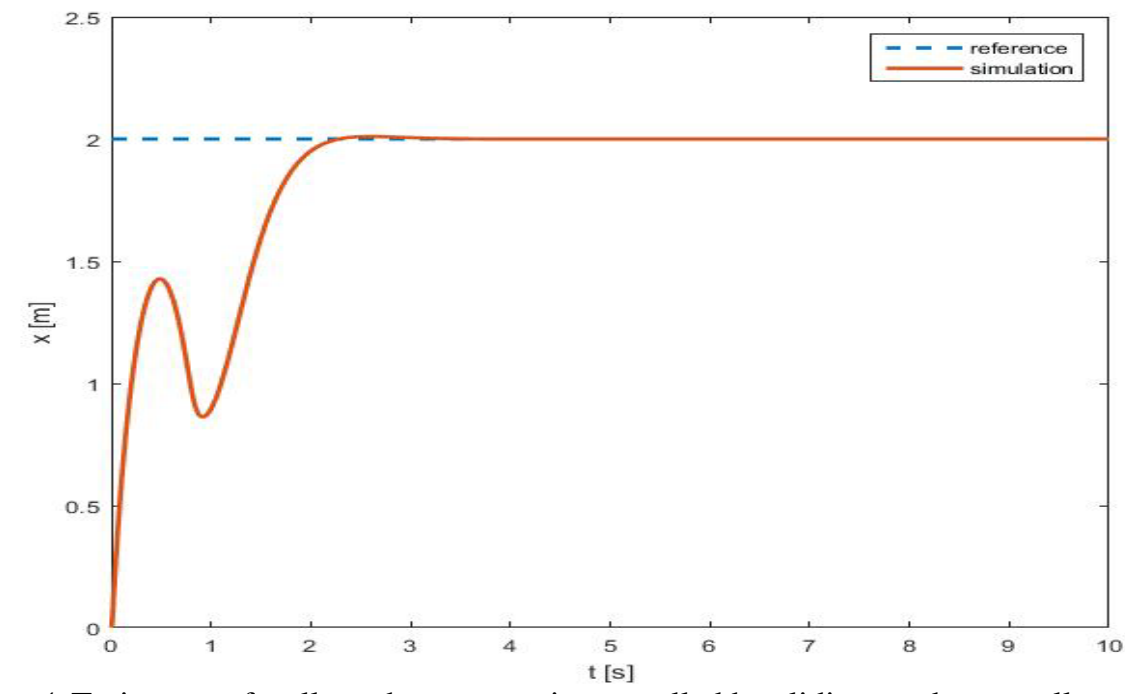

Figure 4. Trajectory of trolley when system is controlled by sliding mode controller without disturbance.

From Figure 4, Figure 5, Figure 6 and Figure 7, it can be seen that ability of resisting disturbance of high order sliding mode controller is very good. But, the quality of swing angle is not good . Although the trolley reaches desired position, but the oscillation of the load is still large.

To fix this problem, RBFNs is used for generating optimal trajectory to reduce sway of load. As shown in the Figure 8 and Figure 9, the trolley reaches desired position and swing angle is very small in the moving process. 


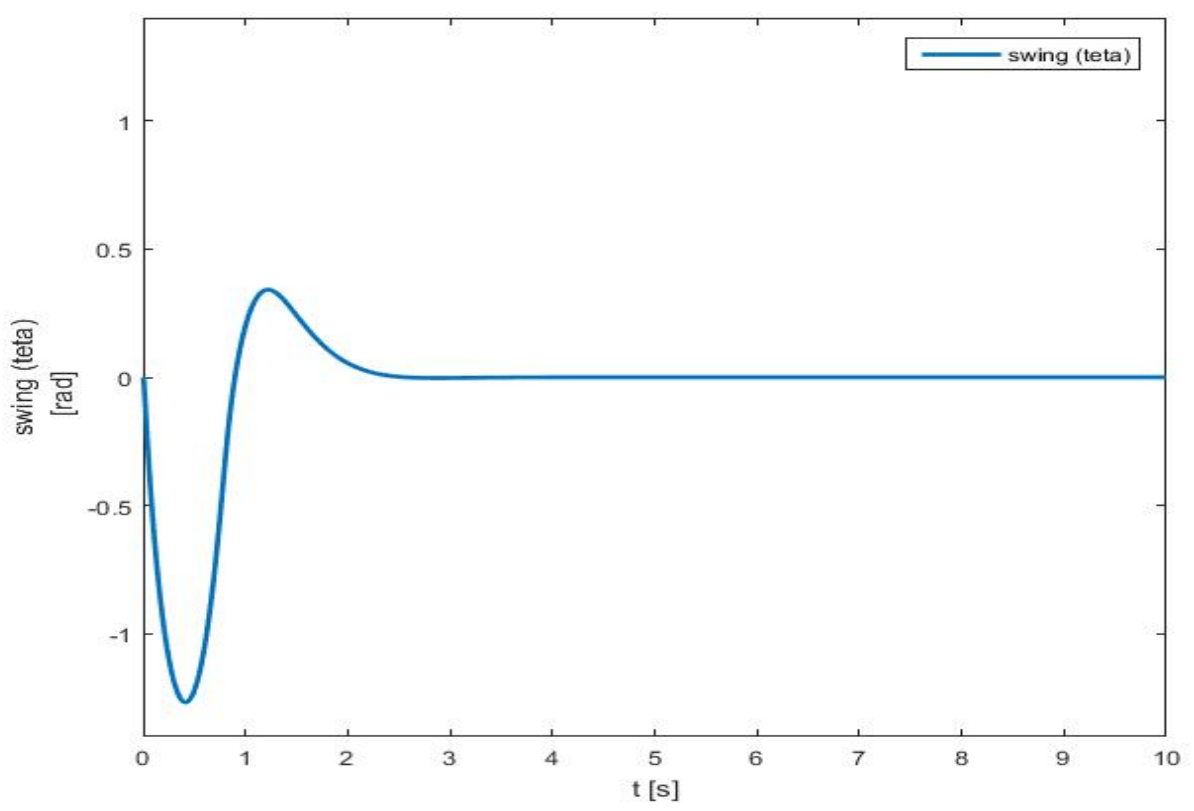

Figure 5. Swing angle of load when system is controlled by sliding mode controller without disturbance.

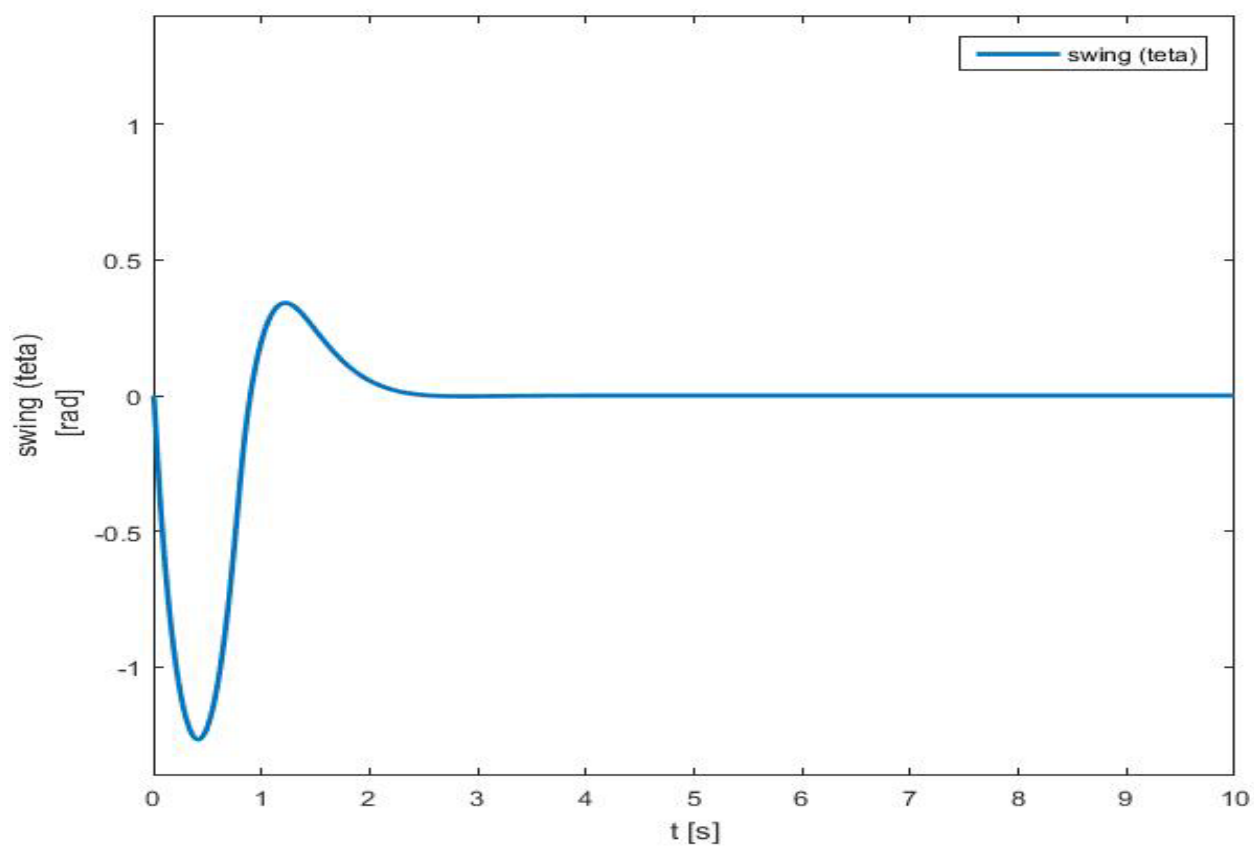

Figure 6. Trajectory of trolley when system is controlled by sliding mode controller with the disturbance at time $1[\mathrm{~s}]$. 


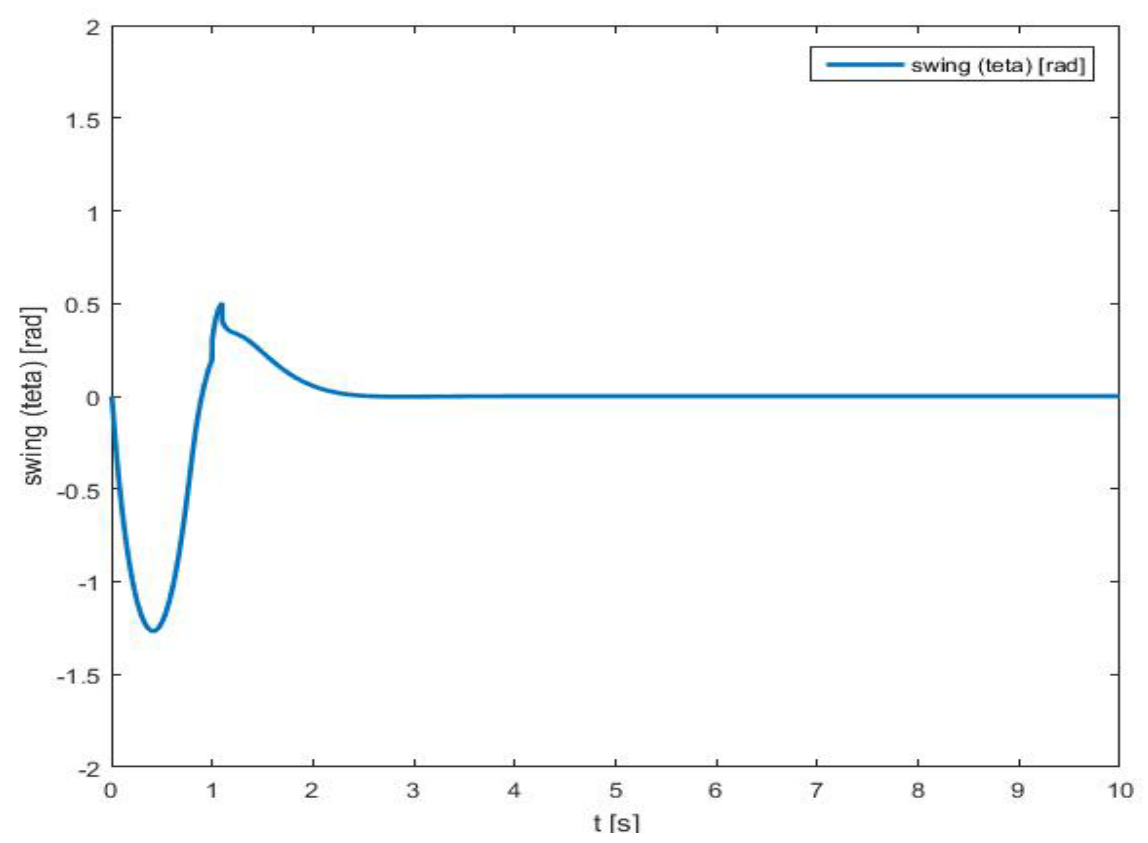

Figure 7. Swing angle of load when system is controlled by sliding mode controller with the disturbance at time $1[\mathrm{~s}]$.

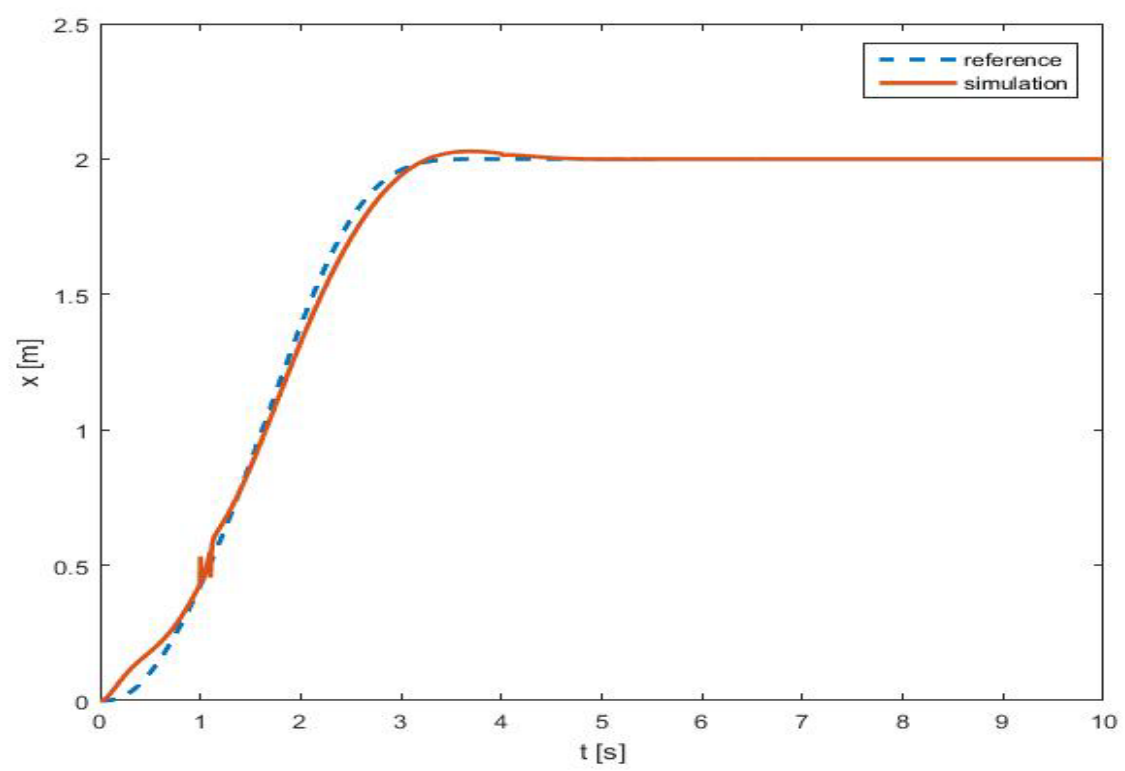

Figure 8. Trajectory of trolley when system is controlled by sliding mode controller combined with compensation anti-sway based on artificial neural network by PSO algorithm with the disturbance at time $1[\mathrm{~s}]$. 


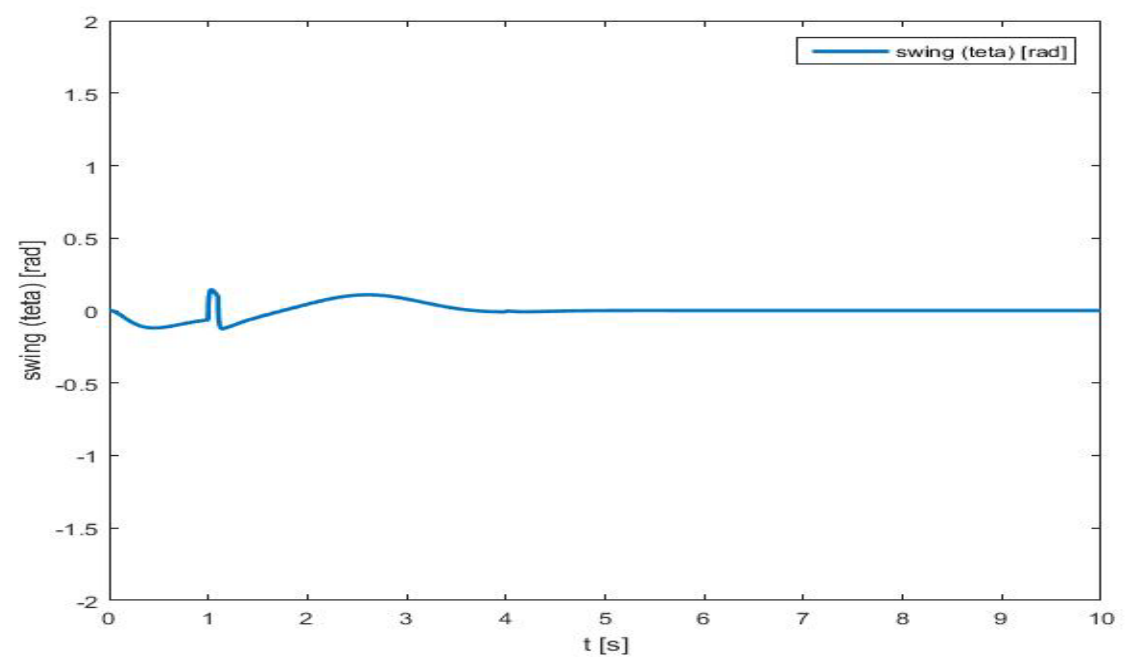

Figure 9. Swing angle of load when system is controlled by sliding mode controller combined with compensation anti-sway based on artificial neural network by PSO algorithm with the disturbance at time $1[\mathrm{~s}]$.

\section{CONCLUSIONS}

In this paper, a new control structure that combines high order sliding mode controller with optimal trajectory set generator is proposed. This scheme ensures that the overhead crane tracks desired trajectory with smaller swing angle of load even under the disturbance condition.

\section{REFERENCES}

1. Jaafar H. I., Z. Mohamed, Abidin A. F. Z., and Ghani Z. A. - PSO tuned PID Controller for a Nonlinear Gantry Crane System, 2012 IEEE International Conference Control System Computer Engineering (ICCSCE), 2012, pp. 515-519.

2. Spong M. - Partial feedback linearization of underactuated mechanical systems, Proceedings of Int., IEEE Conference on Intelligent Robots and Systems Vol. 1, Sep. 1214, 1994, Munich, Germany, pp. 314-32

3. Tuan L. A., Kim G. H. and Lee S. G. - Partial feedback linearization control of the three dimensional overhead crane, The 8th IEEE Int. Conference on Automation Science and Engineering, August 20-24, 2012 Seoul, Korea, pp. 1198-1203.

4. Ahmad M. A., Ismail R. M. T. R. and Ramli M. S. - Input shaping techniques for antisway control of 3D gantry crane system, Proceedings of IEEE Int. Conference on Mechatronics and Automation, China, August 2009, pp. 2876-2888.

5. Ahmad M. A., Ismail R. M. T. R., Ramli M. S., Ghani N. M. A. and Hambali N. Investigation of feedforward techniques for anti-sway control of 3D gantry crane system, Proceedings of IEEE Symposium on Industrial Electronics and Applications, Malaysia, October 2009, pp. 265-270. 
6. Chen W. and Saif M. - Output feeback controller design for a class of MIMO nonlinear systems using high order sliding mode differentiators with application to a laboratory 3D crane, IEEE trans. on Industrial Electronics 55 (11) (2008) 3985-3997.

7. Cho S. K. and Lee H. H. - An anti-swing control of a 3D dimentional overhead crane, Proceedings of the American Control Conference, Chicago (June 2000) pp. 1037-1041.

8. Araki N., Konishi Y. and Ishigaki H. - Unknown Parameter Identification Method using Unscented Kalman Filter for Container Crane System, Proceedings of the 2010 International Conference on Modelling, Identification and Control, Okayama, Japan, July 2010, pp. $254-258$.

9. Chwa D. - Nonlinear tracking control of 3D overhead cranes against the initial swing angle and varidation of payload weight, IEEE trans. on Control and Systems Technology 17 (4) (2009) 876-883

10. Rahman E. A., Nayfed A. H. and Masoud Z. - Dynamics and Control of Cranes: A Review, Journal of Vibration and Control 9 (2003) 863-908.

11. Sano H., Ohishi K., Kaneko T., and Mine H. - Anti-sway crane control based on dual state observer with sensor-delay correction, IEEE International Workshop on Advanced Motion Control, Nagaoka, Japan, 2010.

12. Ismail R. M. T. R., Ahmad M. A., Ramli M. S. and Rashidi F. R. M. - Nonlinear dynamic modelling and analysis of a 3D overhead gantry crane system with system parameters variation, Proceedings of Int. Conference on IJSSST 11 (2) (2009) 9-16.

13. Thuan N. Q. and Vesely V. - Robust decentralized controller design for 3D crane, Proceedings of 18th Int. Conference on Process Control, Slovakia, June 2011, pp. 485-490.

14. Shijie D., Zhi L., Zhidong L. and Xiaotian G. - Study of precise positioning and antiswing for the varying rope length in 3D crane systems based on the combination of partial decoupling and fuzzy control, Proceedings of the 2010 IEEE Int. Conference on Robotics and Biomimetics. December 14-18 2010, Tianjin China, pp. 656-661.

15. Kennedy J. and Eberhart R. - Particle Swarm Optimization, Proceedings of the 1995 IEEE International Conference on Neural Networks, IEEE service center, Piscataway, 1995, pp. 1942-1948.

16. Park M. S., Chwa D. and Hong S. K. - Anti-sway tracking control of overhead cranes with system uncertainty and actuator nonlinearity using an adaptive fuzzy sliding-mode control, IEEE Transaction on Industrial Electronics 55 (11) (2008) 3972-3984. 